\title{
Tratamiento y uso de recursos en salud mental de pacientes sin patología.
}

Treatment and use of resources in mental health of patients without pathology.

\author{
Carolina Lozano Serrano a , Alberto Ortiz Lobo ${ }^{\text {b }}$, Carlos González Juárez ${ }^{\mathrm{c}}$. \\ a, by c ${ }^{\text {Psiquiatra }}{ }^{a}$ CSM Leganés. Instituto Psiquiátrico José Germain. Leganés, Madrid, España. ${ }^{b}$ CSM \\ Salamanca. Hospital La Princesa, Madrid, España ${ }^{c}$ Hospital Severo Ochoa. Leganés, Madrid, España.
}

Correspondencia: Carolina Lozano Serrano (carolinalozser@gmail.com)

Recibido: 16/06/2013; aceptado: 05/09/2013

RESUMEN: Objetivo: Determinar si existen diferencias en el uso de recursos y la actividad terapéutica llevada a cabo con las personas con y sin trastorno mental diagnosticable (códigos Z según CIE-10) en los Servicios de Salud Mental.

Metodología: Diseño: estudio descriptivo comparativo. Ámbito: área sanitaria urbana. Población: todos los pacientes derivados al Centro de Salud Mental $(n=1187)$ durante un año. Variables: se han analizado la indicación clínica, el número de citas y la intervención terapéutica a lo largo de un año de seguimiento. Asimismo, se recogieron también variables sociodemográficas, clínicas y asistenciales mediante la entrevista clínica, el test GHQ-28 y una escala de expectativas, para poder completar el estudio.

Resultados: Los códigos Z son dados de alta en mayor proporción, reciben un tercio de visitas a lo largo de un año, faltan a mayor número de citas y tienen una probabilidad casi 5 veces mayor de abandonar tras la primera consulta que los trastornos mentales. Los pacientes diagnosticados de trastorno mental llegan con más tratamiento pautado desde atención primaria, se les añade más tratamiento, sobre todo si habían llegado sin ello a consulta, si viven solos y en el caso de ser derivados vía normal, que los códigos Z. A los 12 meses también están tomando psicofármacos en mayor proporción.

Conclusiones: La indicación de alta o seguimiento en la primera entrevista, el número de citas y los abandonos discriminan las dos poblaciones mejor que en trabajos previos. Nuestros pacientes tienen más medicación pautada en el momento de la consulta, sobre todo en el caso de los códigos $\mathrm{Z}$, que en los estudios internacionales. No obstante, la intervención farmacológica con los códigos Z suele ir encaminada en su mayoría a suspender los psicofármacos.

PALABRAS CLAVE: Códigos Z, trastornos mentales, uso de recursos, tratamiento farmacológico.
ABSTRACT: Aims: to establish the differences in the use of resorurces and treatment with the people with and without diagnosable mental ( $\mathrm{Z}$ codes according to CIE 10) in a Community Mental Health Center.

Method: Design: comparative descriptive study. Scope: urban area. Population: all patientes referred to de mental health service during a year $(n=1187)$. Variables: clinical indication, number of consults and therapeutic intervention throughout a year of pursuit have been analyzed. Also, were also collected sociodemographic, clinical and health services utilization variables by clinical interview, the test GHQ-28 and a scale of expectations, in order to complete the study.

Results: $\mathrm{Z}$ codes are discharged to a greater extent, receive one-third of visits over a year, missing more citations and have a nearly 5 times more likely to leave after the first consultation that mental disorders. Patients diagnosed as mental disorder come with more prescribed treatment from primary care, further treatment is added, especially if they come without it to see, if they live alone and if they are referred for normal track than Z. codes At 12 months are also taking psychotropic drugs in greater proportion.

Conclusions: The indication of discharge or pursuit in the first interview, the number of consults and drop-outs discriminates the two populations better than in previous works. Our patients have more medication at the moment of the consultation, mainly in the case of the $\mathrm{Z}$ codes, than other studies. However, the pharmacology intervention with $\mathrm{Z}$ codes usually goes directed in its majority to suspend the psychotropic drug.

KEY WORDS: Z codes, mental disorder, use of resources, psychotropic drug treatment. 


\section{Introduccion}

En los últimos años estamos asistiendo al fenómeno de la medicalización de la vida por el cual la medicina está expandiendo sus límites hacia estados o situaciones que antes no se consideraban objeto de atención sanitaria. En el campo de la salud mental, las reacciones emocionales normales en forma de sentimientos legítimos y adaptativos como la tristeza, la rabia, el miedo o la indignación son consideradas con mayor frecuencia, entidades que precisan un tratamiento. Esto supone que muchos pacientes que no pueden ser diagnosticados de trastorno mental (los que la CIE-10 denomina códigos Z) son tratados no solo en atención primaria (AP), sino en los servicios de salud mental y expuestos a los efectos secundarios de los tratamientos que se les prescribe (1-2). Se ha señalado que el tratamiento de los códigos $\mathrm{Z}$ y del disconfort de la vida diaria, no tiene fundamento científico (3), es éticamente cuestionable (4) y daña a los pacientes (5). Además representa un gasto innecesario en los servicios públicos de salud mental y perjudica a los pacientes más graves porque se proporciona mayor atención a quién la demanda y no tanto a quien la necesita por la ley de cuidados inversos (6).

No es sencillo trazar la frontera entre los códigos $\mathrm{Z}$ y los trastornos mentales porque probablemente no la hay. Se trata más bien de un territorio caracterizado por el sufrimiento y el malestar que bordea entre la salud y la enfermedad. Sin embargo, en ningún caso este grupo de pacientes debería ser tratado en un servicio de salud mental especializado porque incluso los trastornos mentales leves ya deberían recibir el tratamiento en atención primaria. Detectar a los códigos Z permite evitar exponer a esta población a la mayor iatrogenia de un servicio especializado y favorece la racionalización de los recursos asistenciales.

Sólo hay cuatro trabajos publicados en la literatura científica que revisan el fenómeno de los pacientes sin trastorno mental diagnosticable y comparan esta población con la de los trastornos mentales de forma escueta y en un sistema sanitario diferente (7-10).

El objetivo de nuestro estudio es determinar si existen diferencias en el uso de recursos y la actividad terapéutica llevada a cabo con las personas con y sin trastorno mental diagnosticable en los Servicios de Salud Mental.

\section{Material y métodos}

Diseño

Estudio descriptivo longitudinal. 
ORIGINALES Y REVISIONES

\section{Emplazamiento}

Este estudio se realizó en el Centro de salud mental (CSM) del Distrito de Salamanca que pertenece al Área Sanitaria 2 de Madrid y atiende a una población urbana de 140.000 habitantes.

\section{Población}

Se incluyeron a todos los pacientes mayores de 18 años derivados al CSM que solicitaron atención durante un año, que no tenían historia clínica abierta o, si la tenían, habían transcurrido más de dos años desde su último contacto en el centro.

La derivación desde AP se realiza cumplimentando un volante por el médico de familia, en el que se especifica si solicita una cita preferente (en menos de dos semanas) o normal (entre uno y dos meses de espera). Por defecto, se cita como normal. Excepcionalmente se reciben derivaciones desde el hospital de referencia, otros servicios médicos especializados, Servicios Sociales del distrito o de los juzgados. Finalmente acudieron a la consulta 1004 pacientes. Se obtuvo una muestra total de 244 pacientes sin trastorno mental diagnosticable. Se seleccionó otra muestra de igual tamaño de pacientes con trastorno mental diagnosticable mediante un muestreo aleatorio simple entre los 760 pacientes diagnosticados de trastorno mental.

\section{Mediciones e intervenciones}

En la primera cita en el CSM (cita de evaluación) previo a la consulta, se invita a los pacientes a participar en el estudio y se solicita su consentimiento informado. Se les entrega el cuestionario GHQ-28 (11-12) para ser autocumplimentado en la sala de espera, el SCL-90-R (Symptom Check List) (13) y una escala de expectativas, tipo likert, puntuada de 0 (nada) a 10 (completamente), en la que se le pregunta al paciente cuánto cree que va a mejorar al venir al centro de salud mental. Se consideraron expectativas negativas a aquellas que puntuaron entre $0 \mathrm{y}$ 4,5 , positivas a las que lo hicieron entre 5 y 8,5 y mágicas las de aquellos pacientes que puntuaron entre 9 y 10 .

A continuación, se realiza una entrevista clínica de una hora realizada por uno de los cinco psiquiatras o dos psicólogos del equipo del CSM según la práctica asistencial habitual. Todos los profesionales involucrados en el estudio tiene una experiencia clínica de al menos de 10 años en la valoración del trastorno mental y en el uso diario de la CIE 10. De todos modos, para incrementar la fiabilidad, previamente se consensuaron los criterios CIE-10, se delimitó qué se consideraba trastorno mental con todos los profesionales implicados y se realizó un estudio piloto durante 3 meses. Tras la evaluación se emitía un juicio diagnóstico y se clasificaba a los pacientes como trastornos mentales o no, según la CIE-10, y se decidía la actitud terapéutica de alta o seguimiento. 
De todos los pacientes se recogían las siguientes variables dependientes: indicación tras evaluación, indicación terapéutica a los 6 y 12 meses de seguimiento, mantenimiento o no del tratamiento inicial pautado desde Atención Primara, tratamiento que se suspende y tratamiento que se añade tras la evaluación, tratamiento farmacológico a los 12 meses de seguimiento y el número de citas y ausencias relativas a lo largo de los 6 y 12 meses de evolución. Se estableció un punto de corte en 6 visitas al centro de salud mental para poder comparar los resultados con otros estudios. Además se recogieron variables sociodemográficas (sexo, edad, estado civil, convivencia, nivel educativo, situación laboral), asistenciales (tipo y sexo del profesional, tipo de derivación) y clínicas (antecedentes psiquiátricos, tratamiento inicial) para poder realizar un análisis estratificado de las variables dependientes.

Para conocer los datos correspondientes a los 6 y 12 meses de seguimiento se consultaron las historias clínicas de los pacientes.

\section{Estrategia de análisis}

Se realizó un análisis comparativo de las dos muestras. Para ello se emplea t de Student para muestras independientes para la comparación de variables cuantitativas y chi cuadrado para variables categóricas. En segundo lugar se realizó un análisis estratificado para las variables que recogen la indicación terapéutica tras la evaluación, la intervención psicofarmacológica en el momento de la entrevista y a los 12 meses y el número de citas y ausencias con el objeto de identificar factores de confusión o modificadores del efecto.

\section{Resultados}

Los códigos $\mathrm{Z}$ son dados de alta con mayor frecuencia en la primera cita y realizan menos seguimientos al cabo de 6 y 12 meses que los trastornos mentales. El seguimiento, cuando se produce, es con mayor frecuencia, psiquiátrico, para los trastornos mentales y psicológico para los códigos Z (Tabla 1).

Los códigos $Z$ tienen pautado con menor frecuencia un tratamiento psicofarmacológico desde atención primaria respecto a los trastornos mentales, pero tienen prescritas más benzodicepinas que estos. Cuando llegan al CSM, se añade tratamiento a un $25 \%$ de los códigos $\mathrm{Z}$ y suele ser benzodiacepinas mientras que a los trastornos mentales se les añade, en un 38\%, principalmente antidepresivos (Tabla 2).

El añadir tratamiento está modulado por el tipo de derivación, el tratamiento inicial y el resultado del GHQ-28 (Tabla 3).

Globalmente no hay diferencias entre códigos $\mathrm{Z}$ y trastornos mentales al modificar tratamiento psicofarmacológico en la primera cita, aunque esta variable está 
Tabla 1.

Indicación terapéutica tras la evaluación y a los 6 y 12 meses.

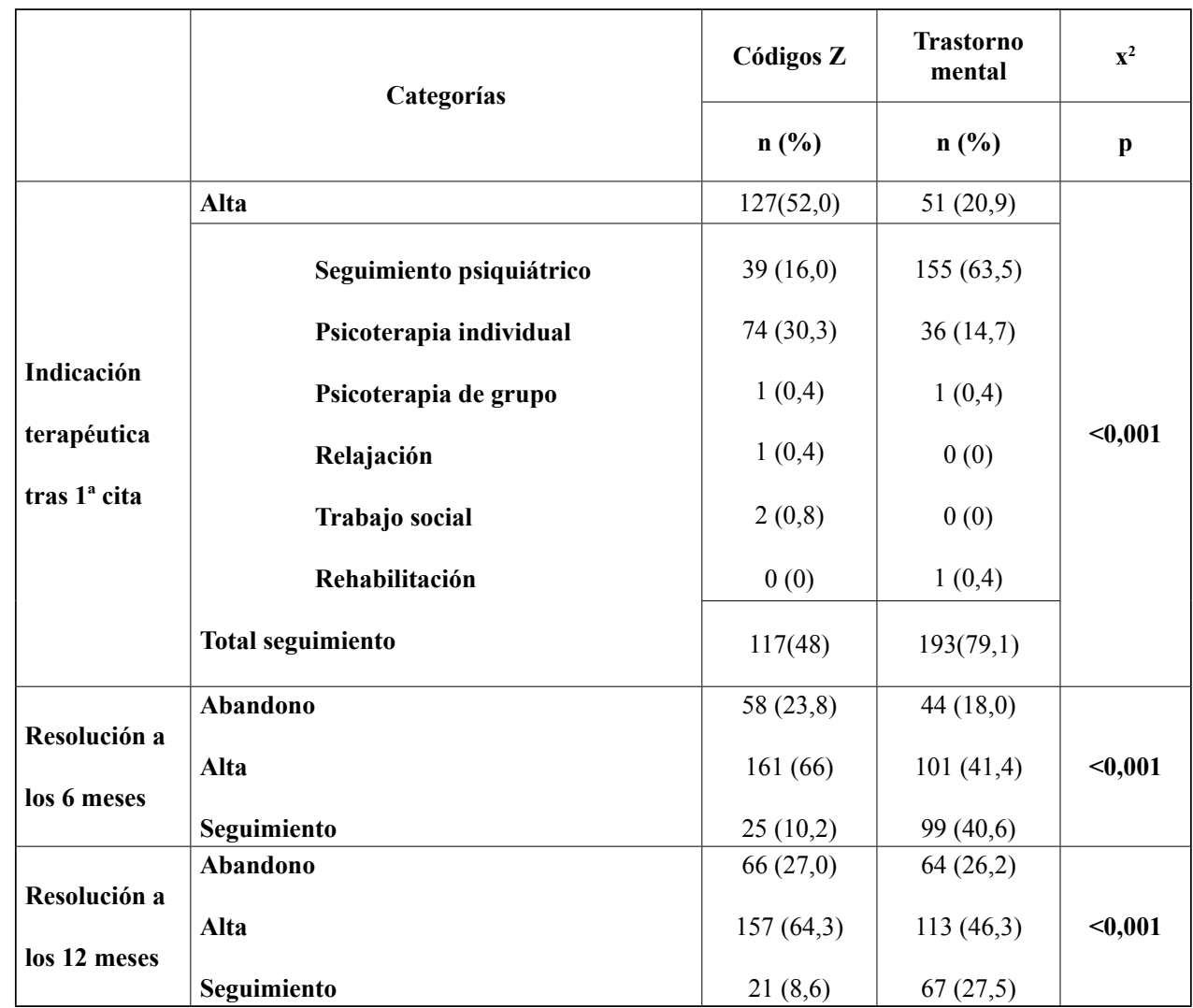

modulada por la convivencia, el tipo de derivación y si tienen tratamiento inicial pautado en AP (Tabla 4).

La suspensión de tratamiento en la primera cita es más frecuente entre los códigos $\mathrm{Z}$ y esta variable está modulada por el tipo de derivación, la convivencia y las expectativas del paciente (Tabla 5).

Al cabo de un año, la mayoría de los códigos $\mathrm{Z}$ no tiene prescrito ningún psicofármaco $(59,3 \%)$ en tanto que la mayoría de los trastornos mentales $(73,6 \%)$ sí que lo tienen pautado (Tabla 2).

A los 6 y 12 meses de la evaluación, los trastornos mentales tienen 1,84 y 
Tabla 2

Frecuencia de intervención terapéutica de las dos poblaciones.

\begin{tabular}{|c|c|c|c|c|}
\hline \multirow{2}{*}{ Variables } & \multirow{2}{*}{ Categorías } & Códigos Z & $\begin{array}{c}\text { Trastorno } \\
\text { mental }\end{array}$ & $\mathrm{x}^{2}$ \\
\hline & & n (\%) & n (\%) & $\mathbf{p}$ \\
\hline \multirow{3}{*}{$\begin{array}{l}\text { Trat a miento } \\
\text { inicial desde AP }\end{array}$} & No & $124(50,8)$ & $98(40,1)$ & \multirow{3}{*}{$<0,001$} \\
\hline & \multirow[t]{2}{*}{$\begin{array}{l}\text { Benzodiacepinas (BDZ) } \\
\text { Antidepresivos (AD) } \\
\text { Benzodiacepinas + antidepresivo } \\
\text { Neurolépticos + - BDZ + - AD } \\
\text { Otros } \\
\text { Total con tratamiento }\end{array}$} & $\begin{array}{c}63(25,8) \\
16(6,6) \\
37(15,2) \\
2(0,8) \\
1(0,4)\end{array}$ & $\begin{array}{c}43(17,6) \\
40(16,3) \\
30(12,3) \\
29(11,8) \\
4(1,6)\end{array}$ & \\
\hline & & $120(49,2)$ & $146(59,9)$ & \\
\hline \multirow{3}{*}{$\begin{array}{l}\text { Trat a miento } \\
\text { suspendido }\end{array}$} & No & $195(79,9)$ & $218(89,3)$ & \multirow{3}{*}{0,005} \\
\hline & \multirow[t]{2}{*}{$\begin{array}{l}\text { Benzodiacepinas } \\
\text { Antidepresivos } \\
\text { Benzodiacepinas+antidepresivo } \\
\text { Neurolépticos + - BDZ + - AD } \\
\text { Otros } \\
\text { Total con tratamiento }\end{array}$} & $\begin{array}{c}20(8,2) \\
20(8,2) \\
7(2,9) \\
2(0,8) \\
0(0)\end{array}$ & $\begin{array}{c}10(4,1) \\
8(3,2) \\
2(0,8) \\
4(1,6) \\
2(0,8)\end{array}$ & \\
\hline & & $49(20,1)$ & $26(10,7)$ & \\
\hline \multirow{3}{*}{$\begin{array}{l}\text { Trat a miento } \\
\text { añadido }\end{array}$} & No & $183(75,0)$ & $153(62,7)$ & \multirow{3}{*}{$<0,001$} \\
\hline & \multirow[t]{2}{*}{$\begin{array}{l}\text { Benzodiacepinas (BDZ) } \\
\text { Antidepresivos (AD) } \\
\text { Benzodiacepinas + antidepresivo } \\
\text { Neurolépticos+ - BDZ + - AD } \\
\text { Otros } \\
\text { Total con tratamiento }\end{array}$} & $\begin{array}{c}52(21,3) \\
3(1,2) \\
6(2,5) \\
0(0) \\
0(0)\end{array}$ & $\begin{array}{c}25(10,2) \\
31(12,7) \\
24(9,8) \\
11(4,5) \\
0(0)\end{array}$ & \\
\hline & & $61(25,0)$ & $91(37,3)$ & \\
\hline \multirow{3}{*}{$\begin{array}{l}\text { Tratamiento a } \\
\text { los } 12 \text { meses* }\end{array}$} & Ninguno & $86(59,3)$ & $23(26,4)$ & \multirow{3}{*}{$<0,001$} \\
\hline & \multirow[t]{2}{*}{$\begin{array}{l}\text { Benzodiacepinas (BDZ) } \\
\text { Antidepresivos (AD) } \\
\text { Benzodiacepinas + antidepresivos } \\
\text { Neurolépticos + - BDZ + - AD } \\
\text { Otros } \\
\text { Total con tratamiento }\end{array}$} & $\begin{array}{c}37(25,5) \\
8(5,5) \\
12(8,3) \\
0(0) \\
2(1,4)\end{array}$ & $\begin{array}{c}7(8,05) \\
10(11,4) \\
23(26,4) \\
19(21,8) \\
5(5,7)\end{array}$ & \\
\hline & & $59(40,7)$ & $64(73,6)$ & \\
\hline
\end{tabular}

* Respecto a 146 códigos $\mathrm{Z}$ que pueden ser reevaluados y 88 de los trastornos mentales de los que se han podido obtener datos por la historia clínica o registro informatizado del centro. El resto de las variables se analiza en el total de la muestra. 
Tabla 3.

Moduladores de la relación entre ser diagnosticado Código $Z$ o trastorno mental y no recibir variación en el tratamiento en la primera cita.

\begin{tabular}{|c|c|c|c|c|c|c|}
\hline & & \multicolumn{2}{|c|}{$\begin{array}{l}\text { No se varia } \\
\text { tratamiento }\end{array}$} & \multicolumn{2}{|c|}{$\begin{array}{c}\text { Se varia } \\
\text { tratamiento }\end{array}$} & \\
\hline & & $\mathbf{n}$ & $\%$ & $\mathbf{n}$ & $\%$ & \\
\hline \multirow{2}{*}{ Viven solos } & Código Z & 34 & 68,0 & 16 & 32,0 & \multirow{2}{*}{$\begin{array}{c}\mathrm{p}=0,050 \\
\mathrm{OR}=2,204(0,995-4,882)\end{array}$} \\
\hline & Trastorno mental & 27 & 49,1 & 28 & 50,9 & \\
\hline \multirow{2}{*}{ Viven acompañados } & Código Z & 119 & 61,3 & 75 & 38,7 & \multirow{2}{*}{$\begin{array}{c}\mathrm{p}=0,448 \\
\mathrm{OR}=0,851(0,562-1,290\end{array}$} \\
\hline & Trastorno mental & 123 & 65,1 & 66 & 34,9 & \\
\hline \multirow{2}{*}{$\begin{array}{l}\text { Derivación } \\
\text { ordinaria }\end{array}$} & Código Z & 128 & 66,3 & 65 & 33,7 & \multirow{2}{*}{$\begin{array}{c}\mathrm{p}=0,222 \\
\mathrm{OR}=1,306(0,850-2,007)\end{array}$} \\
\hline & Trastorno mental & 101 & 60,1 & 67 & 39,9 & \\
\hline \multirow{2}{*}{$\begin{array}{l}\text { Derivación } \\
\text { preferente }\end{array}$} & Código Z & 25 & 49,0 & 26 & 51,0 & \multirow{2}{*}{$\begin{array}{c}\mathrm{p}=0,083 \\
\mathrm{OR}=0,530(0,257-1,091)\end{array}$} \\
\hline & Trastorno mental & 49 & 64,5 & 27 & 35,5 & \\
\hline \multirow{2}{*}{$\begin{array}{l}\text { Sin trat. } \\
\text { inicial }\end{array}$} & Código Z & 98 & 79,0 & 26 & 21,0 & \multirow{2}{*}{$\begin{array}{c}\mathrm{P}<0,001 \\
\mathrm{OR}=3,926(2,184-7,057)\end{array}$} \\
\hline & Trastorno mental & 48 & 49,0 & 50 & 51,0 & \\
\hline \multirow{2}{*}{ Con trat. inicial } & Código Z & 54 & 45,4 & 102 & 69,9 & \multirow{2}{*}{$\begin{array}{c}\mathrm{P}<0,001 \\
\mathrm{OR}=0,358(0,216-0,594)\end{array}$} \\
\hline & Trastorno mental & 65 & 54,6 & 44 & 30,1 & \\
\hline
\end{tabular}

Tabla 4.

Moduladores de la relación entre ser diagnosticado Código Z o trastorno mental y que se añada tratamiento en la primera cita.

\begin{tabular}{|c|c|c|c|c|c|c|}
\hline & & \multicolumn{2}{|c|}{$\begin{array}{l}\text { No se añade } \\
\text { tratamiento }\end{array}$} & \multicolumn{2}{|c|}{$\begin{array}{c}\text { Se añade } \\
\text { tratamiento }\end{array}$} & \\
\hline & & $\mathbf{n}$ & $\%$ & $\mathbf{n}$ & $\%$ & \\
\hline \multirow{2}{*}{ Derivación ordinaria } & Código $\mathrm{Z}$ & 148 & 76,7 & 45 & 23,3 & $\mathrm{p}=0,002$ \\
\hline & Trastorno mental & 103 & 61,3 & 65 & 38,7 & $\mathrm{OR}=2,076(1,316-3,274)$ \\
\hline \multirow{2}{*}{$\begin{array}{l}\text { Derivación } \\
\text { preferente }\end{array}$} & Código $\mathrm{Z}$ & 16 & 31,4 & 35 & 68,6 & $\mathrm{p}=0,739$ \\
\hline & Trastorno mental & 26 & 34,2 & 50 & 65,8 & $\mathrm{OR}=1,138(0,533-0,427)$ \\
\hline \multirow{2}{*}{$\begin{array}{l}\text { Sin trat. } \\
\text { de AP }\end{array}$} & Código $\mathrm{Z}$ & 99 & 79,8 & 25 & 20,2 & $\mathrm{p}<0,001$ \\
\hline & Trastorno mental & 48 & 49,0 & 50 & 51,0 & $\mathrm{OR}=4,125(2,284-7,449)$ \\
\hline \multirow{2}{*}{$\begin{array}{l}\text { Con trat. } \\
\text { de AP }\end{array}$} & Código Z & 83 & 69,7 & 36 & 30,3 & $\mathrm{p}=0,699$ \\
\hline & Trastorno mental & 105 & 71,9 & 41 & 28,1 & $\mathrm{OR}=0,90(0,529-1,533)$ \\
\hline \multirow{2}{*}{ No caso psiquiátrico } & Código $\mathrm{Z}$ & 33 & 91,7 & 3 & 8,3 & $\mathrm{p}=0,23$ \\
\hline & Trastorno mental & 24 & 70,6 & 10 & 29,4 & $\mathrm{OR}=4,5(1,138-18,461)$ \\
\hline \multirow{2}{*}{ Caso psiquiátrico } & Código $\mathrm{Z}$ & 133 & 71,9 & 52 & 28,1 & $\mathrm{p}=0,028$ \\
\hline & Trastorno mental & 129 & 61,4 & 81 & 38,6 & $\mathrm{OR}=1,606(1,051-2,455)$ \\
\hline
\end{tabular}


Tabla 5.

Moduladores de la relación entre ser diagnosticado Código Z o trastorno mental y que se suspenda tratamiento en la primera cita.

\begin{tabular}{|c|c|c|c|c|c|c|}
\hline & \multicolumn{2}{|c|}{$\begin{array}{c}\text { No se } \\
\text { suspende } \\
\text { tratamiento }\end{array}$} & \multicolumn{2}{|c|}{$\begin{array}{l}\text { Se suspende } \\
\text { tratamiento }\end{array}$} & \multirow[t]{2}{*}{$\mathrm{p}$} \\
\hline & & n & $\%$ & n & $\%$ & \\
\hline \multirow{2}{*}{ Derivación ordinaria } & Código Z & 60 & 67,4 & 29 & 32,6 & $\mathrm{p}=0,082$ \\
\hline & Trastorno mental & 68 & 79,1 & 18 & 20,9 & $\mathrm{OR}=0,548(0,277-1,084)$ \\
\hline \multirow{2}{*}{$\begin{array}{l}\text { D e r i v a c i ó n } \\
\text { preferente }\end{array}$} & Código Z & 11 & 36,7 & 19 & 63,3 & $\mathrm{p}<0,001$ \\
\hline & Trastorno mental & 52 & 86,7 & 8 & 13,3 & $\mathrm{OR}=0,089(0,031-0,255)$ \\
\hline \multirow{2}{*}{$\begin{array}{l}\text { Viven } \\
\text { solos }\end{array}$} & Código Z & 20 & 74,1 & 7 & 25,9 & $\mathrm{p}=0,829$ \\
\hline & Trastorno mental & 26 & 76,5 & 8 & 23,5 & $\mathrm{OR}=0,879(0,273-2,832)$ \\
\hline \multirow{2}{*}{ Viven acompañados } & Código Z & 51 & 55,4 & 41 & 44,6 & $\mathrm{P}<0,001$ \\
\hline & Trastorno mental & 94 & 83,9 & 18 & 16,1 & $\mathrm{OR}=0,238(0,124-0,457)$ \\
\hline \multirow{2}{*}{$\begin{array}{l}\text { Expectativas } \\
\text { negativas }\end{array}$} & Código Z & 11 & 78,6 & 3 & 21,4 & $p=0,924$ \\
\hline & Trastorno mental & 12 & 80,0 & 3 & 20,0 & $\mathrm{OR}=0,917(0,152-5,531)$ \\
\hline \multirow{2}{*}{$\begin{array}{l}\text { Expectativas } \\
\text { positivas }\end{array}$} & Código Z & 30 & 60,0 & 20 & 40,0 & $\mathrm{p}<0,018$ \\
\hline & Trastorno mental & 43 & 81,1 & 10 & 18,9 & $\mathrm{OR}=0,349(0,143-0,850)$ \\
\hline \multirow[t]{2}{*}{ Expectativas } & Código Z & 17 & 53,1 & 15 & 46,9 & $\mathrm{p}<0,001$ \\
\hline & Trastorno mental & 35 & 87,5 & 5 & 12,5 & $\mathrm{OR}=0,162(0,050-0,520)$ \\
\hline
\end{tabular}

3,14 citas más que los códigos $\mathrm{Z}$ y faltan a una cita de cada 5, mientras que los códigos $\mathrm{Z}$ faltan a una cita de cada 2,5 al cabo de 12 meses. Este uso de recursos no está modulado por ninguna variable (tabla 6).

Los trastornos mentales de mayor edad y casados han recibido menos citas que los solteros y, paradójicamente, los que puntúan como no caso en el GHQ-28 reciben dos citas más que aquellos que puntúan como caso al cabo de 12 meses (Tabla 7).

Si marcamos un punto de corte en 6 visitas, los trastornos mentales tienen una probabilidad 4 veces mayor que los códigos $Z$ de recibir más citas de seguimiento en el centro de salud mental ( $O R=4,219, \mathrm{p}<0,001)$ y los códigos $\mathrm{Z}$ tienen casi cinco veces más probabilidad de abandonar el tratamiento que los trastornos mentales $(\mathrm{OR}=0,183, \mathrm{p}<0,001)$. 
Tabla 6.

Citas y ausencias relativas a los 6 y 12 meses.

\begin{tabular}{|c|c|c|c|c|c|c|c|}
\hline & \multicolumn{3}{|c|}{ Códigos Z } & \multicolumn{3}{c|}{ Trastorno mental } & \multirow{2}{*}{$\begin{array}{c}\text { *Diferencia entre } \\
\text { medias }\end{array}$} \\
\cline { 2 - 7 } & Media & Mediana & Rango & Media & Mediana & Rango & 1,84 \\
\hline $\begin{array}{c}\text { Citas en 6 } \\
\text { meses }\end{array}$ & 2,32 & 1 & $1-11$ & 4,16 & 1 & $1-24$ & $(1,2-2,3)$ \\
\hline $\begin{array}{c}\text { Citas en 12 } \\
\text { meses }\end{array}$ & 2,97 & 1 & $1-18$ & 6,11 & 3 & $1-38$ & 3,1 \\
\hline $\begin{array}{c}\text { Ausencias } \\
\text { relativas en } \\
\text { 6 m. }\end{array}$ & 0,4 & 0,3 & $0-1$ & 0,2 & 0 & $0-1$ & $(2,2-4,1)$ \\
\hline $\begin{array}{c}\text { Ausencias } \\
\text { relativas en } \\
\text { 12 m. }\end{array}$ & 0,4 & 0,3 & $0-1$ & 0,2 & 0,8 & $0-1$ & 0,233 \\
\hline
\end{tabular}

*Esta es la diferencia entre las medias medido por la $t$ de student con una $\mathrm{p}<0.001$ tanto a los 6 como a los 12 meses.

Tabla 7.

Modulación del número de citas a 12 meses según convivencia y el GHQ-28

\begin{tabular}{|c|c|c|c|c|}
\hline & & Código Z & Trastorno mental & $\mathbf{p}$ \\
\hline \multirow{3}{*}{ Convivencia } & Soltero & 2,8 & 6,7 & \multirow{3}{*}{0,095} \\
\hline & \begin{tabular}{|l|} 
Casado alguna vez \\
\end{tabular} & 3,0 & 5,4 & \\
\hline & Diferencia de medias & 0,2 & 1,3 & \\
\hline \multirow{3}{*}{ GHQ-28 } & Caso psiquiátrico & 2,3 & 5,8 & \multirow{3}{*}{0,046} \\
\hline & No caso psiquiátrico & 3,0 & 7,8 & \\
\hline & Diferencia de medias & 0,7 & 1,9 & \\
\hline
\end{tabular}

\section{Discusión}

\section{Indicación clínica en la primera cita}

Tras ser evaluados en la primera consulta los códigos $\mathrm{Z}$ son dados de alta en mayor proporción (52\%) que los trastornos mentales (21\%). Parece que es el propio diagnóstico el que influye más en que el paciente realice o no seguimiento, ya que ninguna variable sociodemográfica, ni clínica, ni asistencial influye a la hora de hacer una indicación terapéutica.

En el estudio llevado a cabo por Siddique (7) en tres centros de salud mental comunitarios en Canadá, fueron dados de alta el 37\% de los códigos Z y el 32\% de los trastornos mentales. En el estudio holandés de Spinhoven (8) sólo el 11\% de los códigos $\mathrm{Z}$ y el $7 \%$ de los trastornos mentales fueron dados de alta tras la 
primera consulta. Nuestras cifras son más elevadas sobre todo en el alta de los códigos $\mathrm{Z}$. Este dato resulta más llamativo si consideramos que en nuestro sistema no se permite la autoderivación y los pacientes siempre vienen "filtrados" por la Atención Primaria. Por otra parte, existe una diferencia mayor entre las altas dadas a trastornos mentales y códigos $\mathrm{Z}$ en nuestro estudio que la que obtienen el estudio canadiense y holandés. Probablemente las características asistenciales de nuestro CSM, público y con buena continuidad asistencial con AP, favorece la posibilidad de dar altas a los pacientes en los que no queda clara la necesidad de tratamiento o que este se puede llevar a cabo por su médico de familia.

En nuestro estudio, los pacientes que continúan en seguimiento lo realizan con mayor frecuencia con el psicólogo en el caso de los códigos $\mathrm{Z}$ y con el psiquiatra en el caso de los trastornos mentales. En una línea parecida, el estudio de Siddique (7) revela que más del 90\% de los códigos $\mathrm{Z}$ que realizaron seguimiento, se llevó a cabo por un profesional no médico y el $60 \%$ de los seguimientos de los trastornos mentales fueron psiquiátricos. De todas maneras, no hay que olvidar que el seguimiento de los pacientes está muy relacionado con la composición local de los equipos asistenciales y el perfil particular del profesional que ve al paciente.

\section{Situación asistencial al cabo de 12 meses}

$\mathrm{Al}$ cabo de un año, el 56,4\% de los códigos $\mathrm{Z}$ que no fueron dados de alta en la primera cita abandona el tratamiento frente al $33,1 \%$ de los trastornos mentales. Esto tendría que hacernos reflexionar sobre el tratamiento que ofertamos a los códigos $\mathrm{Z}$ que parece que, o bien no se ajusta a sus expectativas o se mantiene innecesariamente cuando ya han mejorado lo suficiente. En el estudio de Spinhoven (8) la tasa de abandonos es de un 13\% para ambos a lo largo de los doce años que valora el estudio. Este dato, junto con la baja tasa que tenían de altas tras la valoración (11\% para los códigos $\mathrm{Z}$ y $7 \%$ para los trastornos mentales) nos indica que los pacientes del estudio holandés realizan y completan tratamiento en su mayoría. En el estudio de Siddique a los 12 meses siguen en tratamiento el 15\% de los códigos $\mathrm{Z}$ y el $23 \%$ de los trastornos mentales frente al 8,6\% de códigos $\mathrm{Z}$ y el $27,5 \%$ de trastornos mentales de nuestro estudio. Parece que nuestra actitud asistencial discrimina más las dos poblaciones que los estudios anteriores, ya que existen diferencias en las altas tras la primera cita, en el porcentaje de seguimientos y en el de abandonos. Tehrani y colaboradores (14) señalan que el abandono del tratamiento está asociado a vivir solo, estar desempleado, ser joven y haber habido cambios en el tratamiento demandado por el paciente en contra de la orientación de su terapeuta. La razón argumentada por los pacientes en cuanto al abandono era la insatisfacción respecto el cuidado y la necesidad de tratamiento, aunque el 30\% de los que abandonaban estaban satisfechos. Concluyen además que los resultados de los estudios no son constantes, que algunos coinciden en la edad y el desempleo 
ORIGINALES Y REVISIONES

como predictores (15-16) y otros no lo hacen y que la metodología y el concepto de abandono es diferente en unos y otros estudios.

\section{Tratamiento farmacológico}

Los códigos $\mathrm{Z}$ tienen tratamiento psicofarmacológico pautado en casi el $50 \%$ de los casos al llegar a consulta, mientras que los pacientes diagnosticados de trastorno mental lo tienen en un $60 \%$. En el estudio canadiense (10) solo un $11 \%$ de los códigos $\mathrm{Z}$ tenía tratamiento pautado en el momento de la evaluación, un $8 \%$ benzodiacepinas sobre todo y un $3 \%$ antidepresivos/neurolépticos. Los trastornos mentales tenían tratamiento en mayor proporción (48\%) y eran benzodiacepinas y neurolépticos/ antidepresivos en un porcentaje parecido (23 y 25\%). La misma diferencia encuentra Spinhoven en su estudio (8), solo el $13 \%$ de códigos Z traen tratamiento farmacológico frente al $49 \%$ de trastornos mentales. En ambos casos predomina el tratamiento con benzodiacepinas (10\% para los códigos $\mathrm{Z}$ y $25 \%$ para los trastornos mentales). Con estos resultados podemos concluir que en nuestro estudio los pacientes llegan con más medicación pautada en el momento de la consulta, sobre todo en el caso de los códigos Z. A diferencia de los otros trabajos, todos los pacientes de nuestro estudio han sido valorados por un médico de atención primaria, lo que favorece la posibilidad de iniciar un tratamiento farmacológico. Ya se ha descrito que alrededor del 50\% de las prescripciones de antidepresivos en atención primaria se realizan a sujetos que no cumplen los requisitos necesarios para establecer un diagnóstico psiquiátrico (17-19). Por otro lado, nuestro estudio es el más moderno y en los últimos años estamos asistiendo a un gran incremento en la prescripción de tratamientos antidepresivos, propiciado por la enorme popularidad que estos tratamientos han alcanzado actualmente y la propaganda realizada por la industria farmacéutica que obtiene una enorme rentabilidad con las ventas de estos productos (20-23). Unos fármacos cuya eficacia está cada vez más cuestionada, no ya en las reacciones emocionales normales y trastornos adaptativos, sino en los episodios depresivos leves y moderados (24-27).

Respecto al tratamiento psicofarmacológico en el centro de salud mental, tan solo en el estudio de Spinhoven (8) reflejan más uso de psicofármacos entre los trastornos mentales a lo largo del seguimiento, pero no especifican cómo se modifica ni con qué psicofármacos respecto al tratamiento inicial con el que llegan a consulta. En nuestro estudio hemos visto que hay un porcentaje bastante alto, tanto para códigos $\mathrm{Z}$ como para trastornos mentales, a los que se le deja con el mismo tratamiento con el que llegan a consulta. Esta actitud prudente puede explicarse porque el psiquiatra está de acuerdo con el tratamiento pautado por el médico de familia, especialmente si se le va a dar el alta y remitirlo de nuevo a atención primaria, pero también para ver la evolución en los que se quedan en seguimiento.

Respecto a los pacientes a los que se les varía el tratamiento, en el caso de los códigos Z la tendencia es suspender psicofármacos especialmente a los que 
han sido derivados de manera preferente y si viven acompañados probablemente porque se considera que se han tratado con precipitación y cuentan con el apoyo y la contención de la convivencia. En el caso de los trastornos mentales la tendencia es añadirse sobre todo si habían llegado sin tratamiento a consulta, si viven solos y en el caso de ser derivados como normales. La actitud de prescribir psicofármacos en estos pacientes se intensifica quizá porque al ser derivado de esta forma, el malestar pueda ser mayor por el tiempo de evolución y carecen de cierto apoyo al vivir solos.

En nuestro estudio el $23 \%$ de los Z y el $26 \%$ de los trastornos mentales están con tratamiento farmacológico a los 12 meses. Llama la atención en nuestro estudio que el porcentaje de pacientes que sigue con tratamiento a los 12 meses es bastante similar para códigos $\mathrm{Z}$ y para trastornos mentales aunque el perfil sea distinto (benzodiazepinas los primeros y antidepresivos y neurolépticos los segundos). Esta cifra da cuenta de la farmacologización que se realiza de malestares cotidianos que no constituyen un trastorno mental.

\section{Uso de recursos}

El número de citas, menor para los códigos $\mathrm{Z}$, sí que los discrimina frente a los trastornos mentales en nuestro estudio. En los trastornos mentales se dan más citas a los más jóvenes quizá para intentar que el trastorno tenga la menor repercusión posible, y en personas que carecen de la contención o el apoyo de una pareja. Respecto al GHQ-28, que es autoaplicado, el número de citas aumenta si el paciente con trastorno mental puntúa como no caso. Esto puede significar que aquellos pacientes con menor conciencia de enfermedad precisan más citas para poder efectuar una alianza terapéutica.

En el estudio canadiense establecen un punto de corte de 6 visitas y hallaron que el $24 \%$ de los códigos $\mathrm{Z}$ y el $36 \%$ de los trastornos mentales recibían más de 6 visitas. Por otro lado calculaban el tiempo por visita y encontraron que el $27 \%$ de los códigos $\mathrm{Z}$ y el $31 \%$ de los trastornos mentales reciben más de 300 minutos de atención en consulta. Finalmente, no había diferencias significativas en el tiempo total de uso de recursos y este no es un predictor del diagnóstico. En nuestro estudio sí que hay una diferencia significativa entre ambas poblaciones, solo el 11,1\% de los códigos $\mathrm{Z}$ frente al 34,4\% de los trastornos mentales reciben más de 6 visitas en un año.

En el estudio holandés el $46 \%$ de los códigos $\mathrm{Z}$ y el $56 \%$ de los trastornos mentales reciben más de 6 visitas en su tratamiento. En este estudio tanto las tasas de alta tras la evaluación como de abandono son menores que en nuestro trabajo. Esto puede deberse en parte a que proporcionan terapia de pareja o de familia al $36 \%$ de los códigos Z, como una atención específica para buena parte de la problemática de esta población. 
ORIGINALES Y REVISIONES

En el estudio de Figueiredo (9) que compara los códigos Z con problemas familiares frente a los trastornos mentales, el número medio de visitas del total de los pacientes a lo largo de 2 años es de 10. Hay diferencias claras en el uso de recursos de ambas poblaciones, los códigos $\mathrm{Z}$ tienen una media de visitas de 8,3 , y los trastornos mentales con o sin problemas familiares tienen unas medias de visitas de 25,6 y 27,5 respectivamente. Nuestro estudio coincide en encontrar diferencias en ambas poblaciones, pero el número medio de citas en el estudio americano es mayor para ambas poblaciones. Desconocemos si esta diferencia tiene que ver con la organización y estructura del centro sanitario o la forma de trabajar los profesionales (diferencias en la distribución del tiempo de visita, terapias de grupo, etc.).

\section{Conclusiones}

La atención en los centros de salud mental, además de ser eficaz, ha de ser efectiva, eficiente y reducir al mínimo la iatrogenia. Para ello, entre otras cosas, es fundamental discriminar el grupo de personas que no presentan ningún trastorno mental diagnosticable para que no sean expuestos a tratamientos excesivos, innecesarios, perjudiciales y que acarrean un detrimento en la atención a los pacientes que sí se pueden beneficiar de las intervenciones.

Nuestro estudio discrimina en buena medida a estos pacientes ya que los códigos Z, comparados con los trastornos mentales, llegan con menos tratamiento pautado desde AP, se les añade menos tratamiento y se les da con mayor frecuencia el alta en la primera cita. Por otra parte, los códigos $\mathrm{Z}$ faltan más a las citas de seguimiento y abandonan con mayor frecuencia el seguimiento que los trastornos mentales lo que da a entender que esta población se ajusta menos a la atención proporcionada en el CSM.

No es sencillo delimitar la frontera entre los códigos $\mathrm{Z}$ y los trastornos mentales, pero es una tarea importante para frenar la progresiva medicalización de la vida cotidiana que se está produciendo y que lleva a que muchos ciudadanos se expongan a los efectos secundarios de psicofármacos y terapias que se prescriben innecesariamente. Tal vez, frente al dilema cosificador de si el paciente tiene o no un diagnóstico psiquiátrico, la pregunta es si su problema mental se va a beneficiar o no de considerarlo una enfermedad y tributario de un tratamiento. 


\section{BIBLIOGRAFÍA}

(1) Double D. The limits of psychiatry. BMJ 2002; 324:900-4

(2) Ortiz A, García-Moratalla B, Lozano C, De la Mata I, Rodriguez F. Conditions that do not reach the threshold for mental disorder in Spanish psychiatric outpatients: prevalence, treatment and management. Int J of Soc Psychiatry 2011; 57(5): 471-479.

(3) Charlton, B.g. \& McKenzie, K. Treating unhappiness - Society needs palliative psychopharmacology. Br J of Psychiatry 2004; 185: 194-195.

(4) Helmchen H, Linden M. Subthreshold disorders in psychiatry: Clinical reality, methodological artifact and the double-threshold problem. Compr Psychiatry 2000; 41: 1-7.

(5) Ortiz A, Ibáñez I. Iatrogenia y prevención cuaternaria en salud mental. Rev Esp Salud Pública 2011;85(6):513-525.

(6) Hart JT. The inverse care law. The Lancet 1971; 1: 405-412.

(7) Siddique CM, Aubry TD, Mulhall D. The burden of conditions not attributable to mental disorders. Am J Psychiatry 1996; 153: 1489-1491.

(8) Spinhoven P, Van Der Does JW. Conditions not attributable to a mental disorder in Dutch psychiatric outpatients. Psychol Med 1999; 29: 213-220.

(9) De Figueiredo JM, Boerstler H, O’Conell L. Conditions not attributable to a mental disorder: an epidemiologic study of family problems. Am J Psychiatry 1991; 148: 780-783.

(10) Windle C, Thompson JW, Goldman HH, et al. Treatment of patients with no diagnosable mental disorders in CHMCs. Hosp Community Psychiatry 1988; 39: 753-758.

(11) Lobo A, Pérez-Echeverría MJ, Artal J. Validity of the scaled version of the General Health Questionnaire (GHQ-28) in a Spanish population. Psycol Med 1986; 16: 135-140.

(12) Derogatis LR. SCL-90-R, administration, scoring and procedures manual for the revised version. Baltimore: Johns Hopkins University, School of Medicine 1977.

(13) González de Rivera JL, Derogatis LR, De las Cuevas C, et al. The spanish version of the SCL-90-R. Normative data in the general population. Towson: Clinical Psychometric Research 1989.

(14) Tehrani E, Krussel J, Borg L, Munk-Jorgensen P. Dropping out of psychiatric treatment: a prospective study of a first-admission cohort. Acta Psychiatr Scand 1996; 94: 266-271.

(15) Molnar G, Pinchoff DM. Factors in patient elopements from an urban state hospital and strategies for prevention. Hosp Community Psychiatry 1993; 44: 791-792.

(16) Kline J, King M. Treatment dropouts from a community mental health centre. J Com Ment Health J. 1973; 9: 354-360.

(17) Pini S, Tansella M. Detección y manejo de la enfermedad mental en los centros de atención primaria. En: Vázquez-Barquero JL. Psiquiatría en atención primaria. Madrid, Grupo Aula Médica, 1998.

(18) R. Mojtabai, M. Olfson. Proportion of antidepressants prescribed without a psychiatric diagnosis is growing. Health Affairs2011; 30 (8): 1434 DOI: 10.1377/hlthaff.2010.1024

(19) Ramin Mojtabai. The public health impact of antidepressants: An instrumental variable analysis. J Affect Disord, 2011; DOI: 10.1016/j.jad.2011.05.037

(20) Lozano C, Ortiz A. El incremento en la prescripción de antidepresivos. ¿Una panacea demasiado cara? Rev Asoc Esp Neuropsiquiatría 2004; 83-96. 
ORIGINALES Y REVISIONES

(21) Ortiz A, Lozano C. El incremento en la prescripción de antidepresivos. Aten Primaria 2005; 35 (3): 125-5.

(22) Jirón M, Machado M, Ruiz I. Consumo de antidepresivos en Chile entre 1992 y 2004 Rev Med Chile 2008; 136: 1147-1154.

(23) Spence D. Are antidepressants overprescribed? Yes. BMJ, 2013; 346 (jan22 3): f191 DOI: $10.1136 / \mathrm{bmj} . \mathrm{f1} 91$

(24) Turner EH, Matthews AM, Linardatos E, Tell RA, Rosenthal R. Selective publication of antidepressant trials and its influence on apparent efficacy. N Engl J Med 2008; 358(3): 252-260.

(25) Kirsch I, Deacon BJ, Huedo-Medina TB, Scoboria A, Moore TJ, JohnsonBT. Initial severity and antidepressant benefits: a meta-analysis of data submitted to the Food and Drug Administration. Plos Med 2008; 5(2):e45

(26) Fournier JC, DeRubeis RJ, Hollon SD, Dimidjian S, Amsterdam JD, Shelton RC, Fawcett J. Antidepressant Drug Effects and Depression Severity. A Patient-Level Meta-analysis. JAMA 2010; 303(1):47-53. doi:10.1001

(27) Barbui C, Cipriani A, Patel V, Ayuso-Mateos JL, van Ommeren M. Efficacy of antidepressants and benzodiazepines in minor depression: systematic review and meta-analysis. $\mathrm{Br} \mathrm{J}$ Psychiatry 2011; Jan; 198(1):11-6. 